\title{
AMPLITUDE OF WRINKLES IN THIN MEMBRANES
}

\author{
Y.W. Wong and S. Pellegrino \\ Department of Engineering, University of Cambridge \\ Trumpington Street, Cambridge, CB2 1PZ, U.K. \\ pellegrino@eng.cam.ac.uk
}

\begin{abstract}
This paper presents a theory for predicting the wavelength and amplitude of the wrinkles that form in a membrane in pure shear. Predictions from this theory are compared to experimental measurements and a finite element simulation and are found to be very accurate.
\end{abstract}

Keywords: Membrane, Wrinkles, Gossamer spacecraft

\section{INTRODUCTION}

A number of space missions will require high-accuracy membrane structures acting as sunshields, solar arrays and solar sails, radars, and inflatable antennas. These prestressed membrane structures will have to remain partially wrinkled in their operational configuration. Removing the wrinkles would require a biaxially tensile stress state, thus significantly increasing the loads transmitted to the edge deployable structure that supports the membrane.

Wrinkles can reduce the performance of reflectors and sunshields, or cause difficulties in maneuvering solar sails. Hence, it is now important to predict details of the wrinkles, such as wavelength and amplitude, in order to determine if the membrane structures meet the requirements of each particular application.

The wrinkling of membranes has attracted much interest in the past, starting from the observation that the thin-walled shear web of a beam can carry loads well above the initial buckling value; which prompted the development of tension field theory by Wagner (1929). Simpler formulations of this theory by Reissner (1938), Stein and Hedgepeth (1961), and Mansfield $(1969,1989)$ made it possible to find solutions to problems in which the tension-lines are non parallel. Further generalizations were 
proposed by Pipkin (1986) and, more recently, Epstein and Forcinito (2001).

A premise common to all of these formulations, and also to the accompanying numerical solutions that have been pursued alongside, is that the membrane is modelled as a no-compression, two-dimensional continuum with negligible bending stiffness. See Jenkins and Leonard (1991) for an extensive list of references and Adler (2000) for a more recent perspective; see also Liu et al. (2000) for a numerical study in which wrinkle amplitudes were predicted. Hence, it is assumed in effect that an infinite number of wrinkles of infinitesimally small amplitude will form. This is not, of course, what actually happens and it is well known that, although the stress fields produced by these theories are essentially correct, the out-of-plane displacements are significantly different from those observed in practice. This is not a problem in the design of membranes that are purely load-carrying elements, but it is not acceptable in the present context.

The only study, to our knowledge, which has taken into account the role played by the membrane bending stiffness is Rimrott and Cverko's (1986) study of the "hanging blanket" problem. These authors considered a vertical membrane subject to in-plane gravity loading and supported on two level points, a problem for which the tension field solution in terms of cosine-shaped wrinkles had already been determined by Mansfield (1981). However, it can be readily verified — e.g. with a bath towel - that in practice there is only a small number of tension strips, which are in equilibrium in a strongly three-dimensional configuration. Rimrott and Cverko showed that the introduction of a critical, compressive stress perpendicular to the wrinkles produced trends matching the experimentally observed variation of the number of tension strips with the density of the membrane.

In this paper we return to the simple configuration considered in the earliest study of wrinkled membranes, namely the rectangular membrane in shear. For this problem we compute a series of reference solutions, by means of non-linear finite element analysis, where the membrane is accurately modelled with a fine mesh of thin-shell elements. These solutions provide an insight into the stress distribution in the wrinkled membrane, on which we then base a simple analytical solution inspired by Rimrott and Cverko's approach to the hanging blanket. Thus, we obtain predictions for the wrinkle wavelength and amplitude as functions of the angle of shear and of the geometric and material properties of the membrane.

This theory has three key components. First, it is assumed that the tension lines, i.e. the direction of the wrinkles, are determined by a 
standard tension field approach. Second, the (small) compressive stress in the direction perpendicular to the wrinkles is set equal to a critical buckling stress which is a known function of the wavelength of the wrinkles. Third, the stress components along and perpendicular to each wrinkle - modelled as a doubly-curved shell - are in equilibrium in the out-of-plane direction. Results from this model are compared to both direct experimental measurements and finite element simulations, and are found to be in good agreement.

\section{PRELIMINARY CONSIDERATIONS}

Figure 1 shows a photograph of an aluminized Kapton membrane, of length $L=380 \mathrm{~mm}$, height $H=128 \mathrm{~mm}$, and thickness $t=25 \mu \mathrm{m}$, that is attached to rigid blocks along the top and bottom edges and is free along the sides. A relative horizontal displacement $\delta=3 \mathrm{~mm}$ has been imposed between the two blocks without changing their distance, thus imposing a geometric shear strain $\gamma_{g}=2.3 \%$ on the membrane.

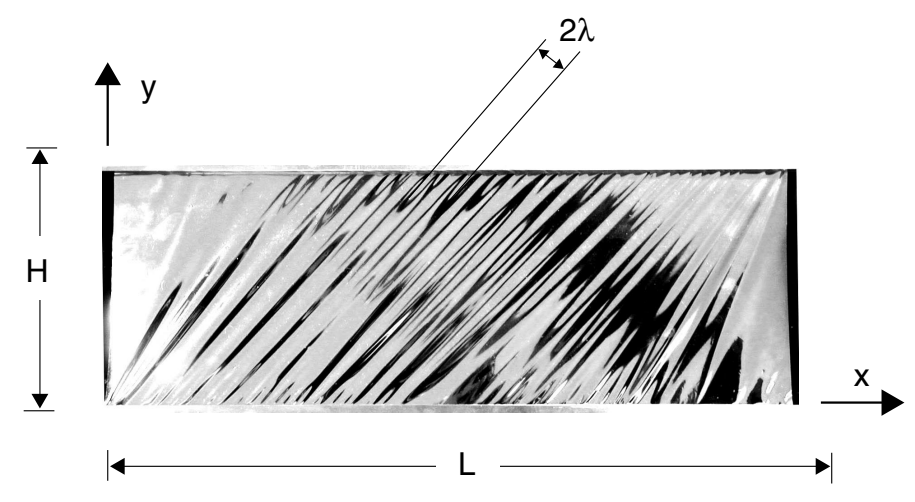

Figure 1. Sheared Kapton sheet, showing extensive wrinkling.

The photograph shows that, instead of deforming purely in two-dimensional shear, the membrane has buckled out of plane, forming a series of approximately straight wrinkles. Excluding the wrinkles originating near the bottom-left and top-right corners, the wrinkles in the central part of the membrane are parallel and uniformly spaced. The wrinkle wavelength, i.e. the distance between two consecutive crests, or troughs, is denoted by $2 \lambda$.

We have carried out a finite-element simulation of the shearing process, using the finite-element package ABAQUS (2000) where the membrane was modelled by four-node thin shell elements (type S4R5). The 
Table 1. Kapton membrane properties.

\begin{tabular}{lc}
\hline Length, $L(\mathrm{~mm})$ & 380 \\
Height, $H(\mathrm{~mm})$ & 128 \\
Thickness, $t(\mathrm{~mm})$ & 0.025 \\
Young's Modulus, $E\left(\mathrm{~N} / \mathrm{mm}^{2}\right)$ & 3530 \\
Poisson's ratio, $\nu$ & 0.3 \\
\hline
\end{tabular}

numerical singularities that occur at the beginning of the analysis, due to the small bending stiffness of the shell elements, were avoided by applying a small, uniform initial stress in the $y$-direction to the membrane and by carrying out an initial, geometrically non-linear step. This was followed by a prediction of the possible wrinkling modes, made by running an eigenvalue buckling step. These modes were superposed and amplified, and applied as initial geometric imperfections, before running the final step. The final analysis of the wrinkled membrane was facilitated by using the automated "stabilize" option in ABAQUS, which introduces dynamic pseudo-inertia and pseudo-damping forces when an instability is detected, thus triggering a pseudo-time integration of the dynamic snap of the system. The default ABAQUS values for the amount of damping that is introduced in the calculation were tuned down to achieve the greatest possible accuracy.

A single type of membrane, made of Kapton, was used for all the studies presented in this paper (except that photographed in Figure 1); its properties are given in Table 1.

Figure 2 is a plot of the principal stresses in a heavily wrinkled membrane, predicted by ABAQUS. For each element we have plotted two vectors aligned with the principal stress directions and of length proportional to the principal stresses. Note that: (i) only the major stress $\sigma_{1}$ is visible in the plot, as the minor stress is very small; (ii) in the central part of the membrane the major principal stress is inclined at $45^{\circ}$; and (iii) there are stress concentrations by a factor of up to 2.5 in the top-left and bottom-right corners.

The same results are also found from tension field theory (Mansfield, 1969) and it is interesting to compare the loci of the crests and troughs predicted with ABAQUS, Figure 3(a), with the tension lines for a semiinfinite strip. Thus, Figure 3(b) shows the tension lines at $5^{\circ}$ intervals as well as the first six wrinkles (a-f) predicted by ABAQUS. It can be seen that wrinkles (a-d), forming respectively at $63^{\circ}, 56^{\circ}, 51^{\circ}$, and $46^{\circ}$ to the horizontal, practically coincide with the corresponding tension lines. 


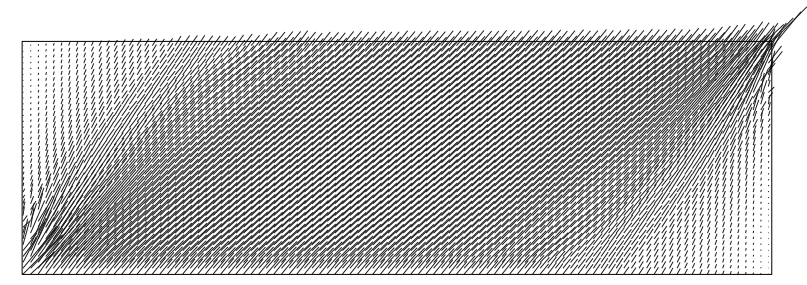

Figure 2. Stress trajectories from ABAQUS solution.

The $45^{\circ}$ tension line is at infinity; but the actual wrinkles are inclined at practically $45^{\circ}$ at only a short distance from the corner.

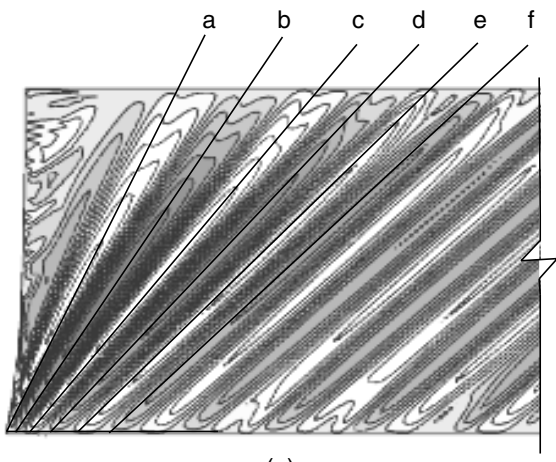

(a)

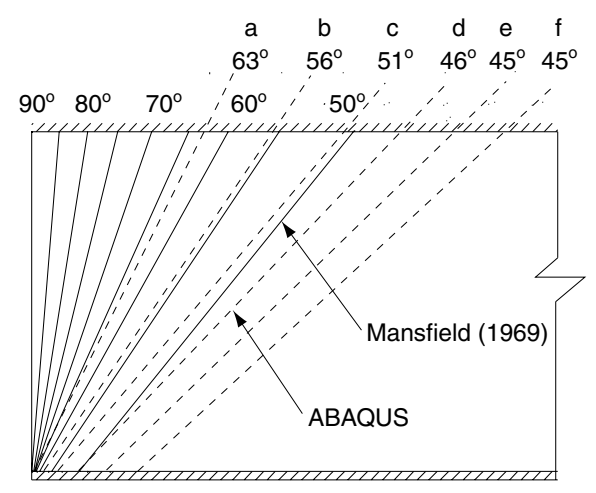

(b)

Figure 3. Comparison between (a) ABAQUS solution and (b) tension field.

Figure 4 is a plot of the distribution of the principal mid-surface stresses across the centre of the membrane, for two different shear displacements. Near the free edges, $\sigma_{1}$ rapidly increases to an approximately uniform, positive value. $\sigma_{2}$ is also essentially uniform in the central region and, although it appears to be zero from this plot, in fact it is negative, albeit small.

The value of the minor principal mid-surface stress at a representative point in the middle of the membrane has been plotted in Figure 5 as a function of the shear displacement $\delta$. The behaviour shown here is characteristic of the post-wrinkled response of the membrane, and it provides a series of important clues. The fact that the mid-surface stress - which of course does not include bending effects - is compressive in 


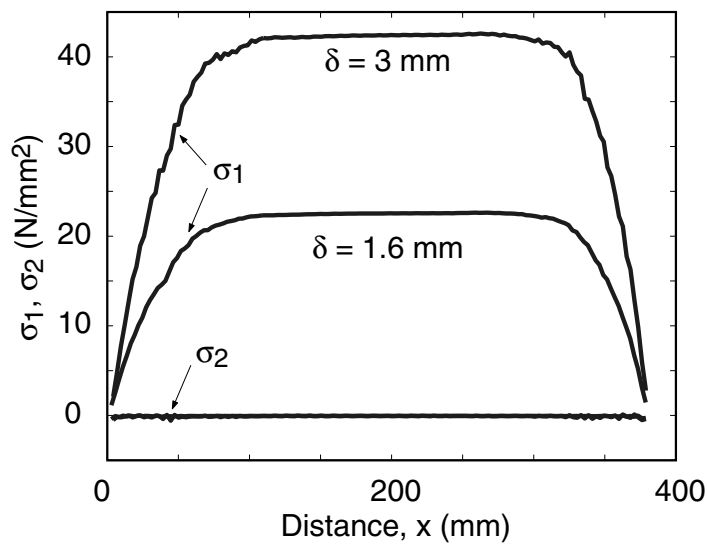

Figure 4. Mid-surface principal stresses through horizontal section at mid-height.

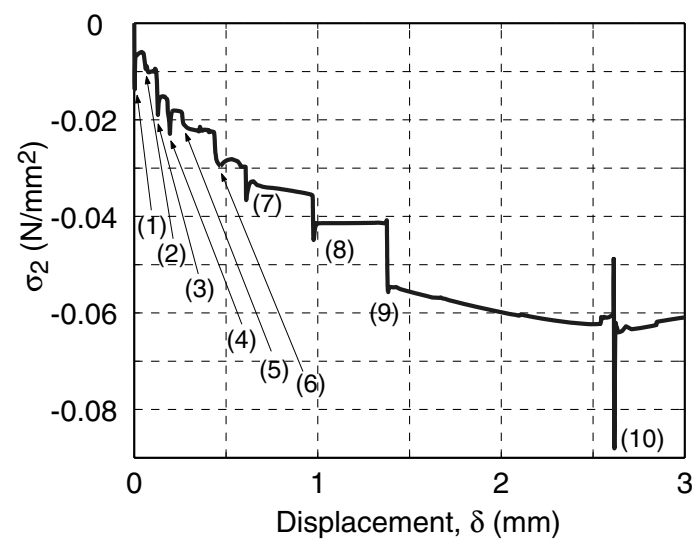

Figure 5. Variation of minor principal stress with shear displacement, at the centre of the membrane.

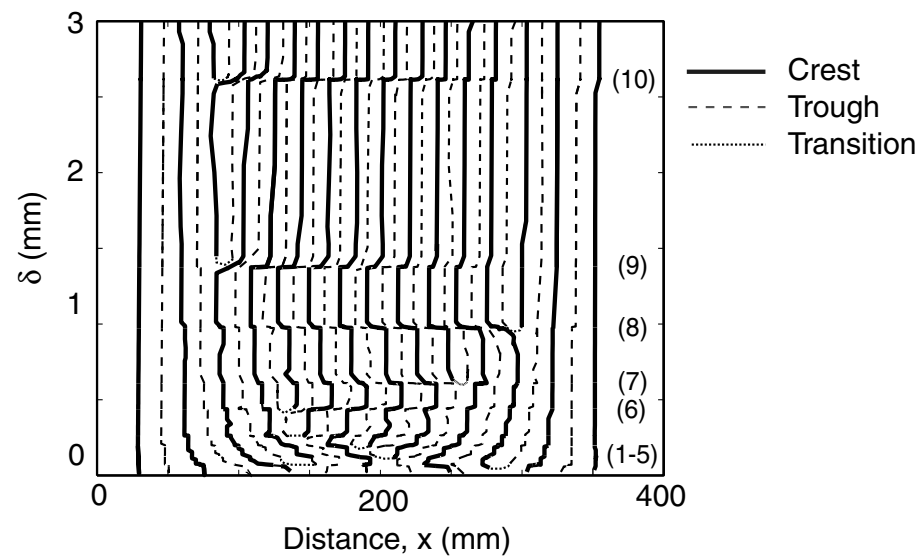

Figure 6. Trajectories of maximum and minimum out-of-plane displacement at mid-height, for increasing shear displacement. 


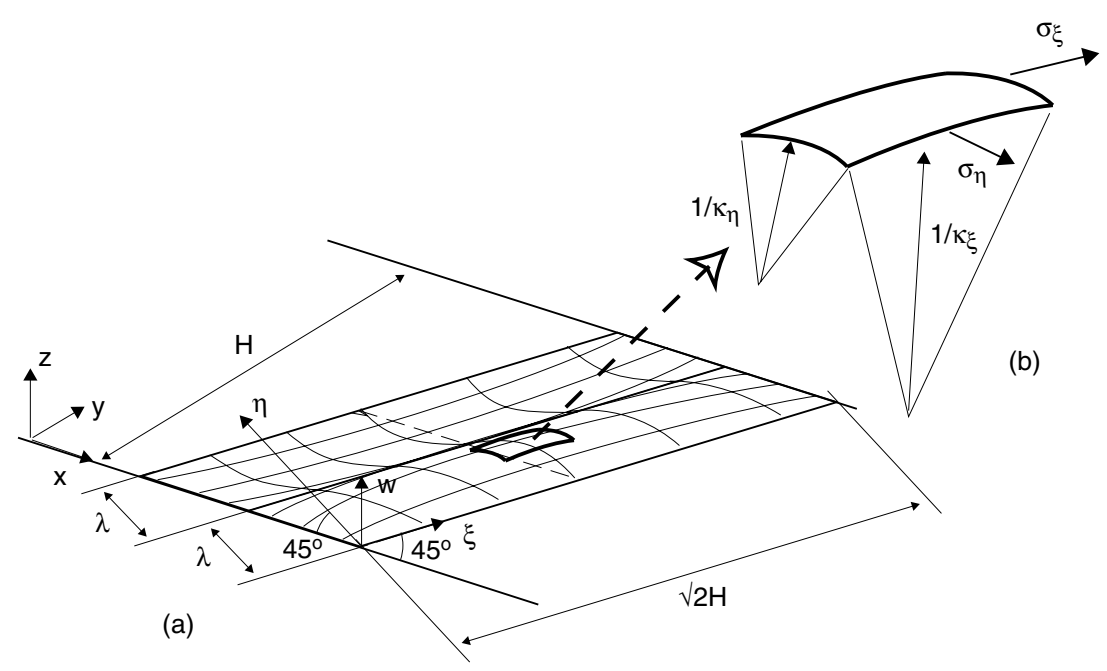

Figure 7. (a) Perspective view of a single wrinkle and (b) infinitesimal free body.

the direction perpendicular to the wrinkles is particularly significant. It is also significant that its value increases with the shear displacement.

This variation has an underlying, square-root type trend, superposed onto a series of sharp peaks and jumps, which correspond to sudden increases in the numbers of wrinkles and a rearrangement of the shape of the central part of the membrane. The complete sequence of the formation of wrinkles at mid-height is shown in Figure 6, where each bifurcation point - and the corresponding "transition" in the plotcorresponds to a jump in Figure 5. Note that the side wrinkles do not move, as they are "pinned" by the corner supports.

\section{ANALYTICAL MODEL}

Consider one of the wrinkles in the central, uniformly wrinkled part of the membrane. From standard tension field theory, its direction is found to be at $45^{\circ}$ to the edges of the membrane, as shown in Figure 7(a).

The initially flat membrane has deformed into a doubly-curved shape lying alternately above and below the original, $x y$ plane of the membrane. This wrinkled surface intersects the $x y$ plane at a regular halfwave distance $\lambda$ and it is observed in practice that these intersections are along straight lines.

A simple mode-shape describing the wrinkled surface can be readily set up using the coordinate system $\xi, \eta$ shown in Figure 7(a). Note that $\xi$ is parallel to the wrinkle direction, and $\eta$ is perpendicular to it. The 
out-of-plane deflection is

$$
w=A \sin \frac{\pi(\xi+\eta)}{\sqrt{2} H} \sin \frac{\pi \eta}{\lambda}
$$

and the in-plane deflection is small. Here, $A$ is the amplitude and $\lambda$ is the half-wavelength, both unknown. Since the wrinkles are long and narrow, $\eta<<\xi$ apart from a small region near the origin. Hence, the mode-shape can be simplified to

$$
w=A \sin \frac{\pi \xi}{\sqrt{2} H} \sin \frac{\pi \eta}{\lambda}
$$

The compressive stress $\sigma_{\eta}$ acting perpendicular to the wrinkles is set equal to the stress required to buckle a simply-supported, infinitely wide plate of length $\lambda$ which is loaded length-wise. Hence, by Euler's formula (Calladine, 1983)

$$
\sigma_{\eta}=-\frac{\pi^{2} E t^{2}}{12\left(1-\nu^{2}\right) \lambda^{2}}
$$

This is very small in comparison with the longitudinal (tensile) stress $\sigma_{\xi}$ and hence the stress-strain relationship for a uniaxial stress state can be assumed to be valid

$$
\sigma_{\xi}=E \epsilon_{\xi}
$$

Since the wrinkles are at $45^{\circ}$ to the $x$ and $y$ axes

$$
\epsilon_{\xi}=\gamma / 2
$$

where $\gamma=\delta / H$ if the strain/stress variation across the wrinkle is neglected. Substituting Equation 5 into Equation 4

$$
\sigma_{\xi}=E \gamma / 2
$$

For equilibrium of the membrane in the normal direction

$$
\sigma_{\xi} \kappa_{\xi}+\sigma_{\eta} \kappa_{\eta}=0
$$

where the principal curvatures are given by

$$
\begin{aligned}
\kappa_{\xi} & =-\frac{\partial^{2} w}{\partial \xi^{2}}=\frac{\pi^{2} A}{2 H^{2}} \sin \frac{\pi \xi}{\sqrt{2} H} \sin \frac{\pi \eta}{\lambda} \\
\kappa_{\eta} & =-\frac{\partial^{2} w}{\partial \eta^{2}}=\frac{\pi^{2} A}{\lambda^{2}} \sin \frac{\pi \xi}{\sqrt{2} H} \sin \frac{\pi \eta}{\lambda}
\end{aligned}
$$

Substituting Equations 3, 6, 8 and 9 into Equation 7, simplifying and rearranging we obtain the following expression for the half-wavelength $\lambda$

$$
\lambda=\frac{\sqrt{\pi}}{\left[3\left(1-\nu^{2}\right)\right]^{\frac{1}{4}}} \frac{\sqrt{H t}}{\gamma^{\frac{1}{4}}}
$$


Next, we obtain an expression for the amplitude $A$ of the wrinkle. This must be such that the contraction of the membrane in the $\eta$-direction

$$
\epsilon_{\eta}=-\gamma / 2
$$

matches the sum of the strain in the membrane

$$
\epsilon_{\eta M}=-\frac{\nu}{E} \sigma_{\xi}
$$

plus the geometric strain produced by the out-of-plane deformation of the membrane, which at mid-height can be shown to have the expression

$$
\epsilon_{\eta G} \approx-\frac{\pi^{2} A^{2}}{4 \lambda^{2}}
$$

Hence,

$$
-\frac{\gamma}{2}=-\frac{\nu}{E} \sigma_{\xi}-\frac{\pi^{2} A^{2}}{4 \lambda^{2}}
$$

Substituting Equation 6 and solving for $A$ gives

$$
A=\frac{\sqrt{2(1-\nu) \gamma}}{\pi} \lambda
$$

from which $\lambda$ can be eliminated using Equation 10, to find

$$
A=\frac{\sqrt{2(1-\nu)}}{\sqrt{\pi}\left[3\left(1-\nu^{2}\right)\right]^{\frac{1}{4}}} \sqrt{H t} \gamma^{\frac{1}{4}}
$$

\subsection{Alternative Formulation}

An alternative approach is to find $\lambda$ by minimizing the strain energy in the wrinkled membrane, modelled as a thin plate stretched in the $\xi$-direction and wrinkled in the $\eta$-direction.

The general expression for the bending strain energy per unit area of an initially flat plate that is bent into a cylindrical shape is

$$
U_{b}=\frac{E t^{3}}{24\left(1-\nu^{2}\right)} \kappa_{\eta}^{2}
$$

As $\kappa_{\eta}$ is not constant, see Equation 9 , the average strain energy, $\overline{U_{b}}$, per unit area is obtained from

$$
\overline{U_{b}}=\frac{E t^{3}}{24\left(1-\nu^{2}\right)}\left(\frac{1}{\sqrt{2} H \lambda} \int_{0}^{\sqrt{2} H} \int_{0}^{\lambda} \kappa_{\eta}^{2} d \xi d \eta\right)=\frac{E t^{3}}{24\left(1-\nu^{2}\right)} \frac{\pi^{4} A^{2}}{4 \lambda^{4}}
$$


The strain energy for stretching in the $\eta$-direction is negligible, hence a general expression for the stretching strain energy per unit area is given by

$$
U_{s}=\frac{1}{2} E t \epsilon_{\xi}^{2}
$$

Here, $\epsilon_{\xi}$ is the sum of the strain due to the in-plane shear, Equation 5, plus that due to the out-of-plane deflection due to wrinkling

$$
\epsilon_{\xi}=\frac{\gamma}{2}+\frac{1}{2}\left(\frac{\partial w}{\partial \xi}\right)^{2}
$$

Thus, $\epsilon_{\xi}$ also is not constant, and hence the average strain energy of stretching, $\overline{U_{s}}$, over a wrinkle is given by

$$
\begin{aligned}
\overline{U_{s}} & =\frac{1}{2} E t\left(\frac{1}{\sqrt{2} H \lambda} \int_{0}^{\sqrt{2} H} \int_{0}^{\lambda} \epsilon_{\xi}^{2} d \xi d \eta\right) \\
& \approx \frac{E t}{2} \frac{\gamma^{2}}{4}+\frac{E t}{2} \frac{\pi^{2} A^{2} \gamma}{16 H^{2}}
\end{aligned}
$$

The first of these terms is independent of the wrinkle amplitude $A$, and hence will not be carried through to the next stage of the analysis.

Hence, the total mean strain energy of wrinkling per unit area (neglecting the term without $A$ ) is given by

$$
\bar{U}=\overline{U_{b}}+\overline{U_{s}}=\frac{E t^{3}}{24\left(1-\nu^{2}\right)} \frac{\pi^{4} A^{2}}{4 \lambda^{4}}+\frac{E t}{2} \frac{\gamma^{2}}{4}+\frac{E t}{2} \frac{\pi^{2} A^{2} \gamma}{16 H^{2}}
$$

Next, we substitute Equation 15 - thus expressing $A$ in terms of $\lambda$ and obtain

$$
\bar{U}=\frac{(1-\nu) E t \gamma}{2}\left(\frac{\pi^{2} t^{2}}{24\left(1-\nu^{2}\right) \lambda^{2}}+\frac{\gamma \lambda^{2}}{8 H^{2}}\right)
$$

which is minimised by differentiating with respect to $\lambda$. This gives

$$
\lambda^{4}=\frac{\pi^{2} t^{2} H^{2}}{3\left(1-\nu^{2}\right) \gamma}
$$

Equations 10 and 24 are equivalent, which shows that the simple equilibrium formulation with an assumed stress $\sigma_{\eta}$ captures the same effects as the energy formulation.

\section{VALIDATION OF THEORY}

We have tested the accuracy of our theory against (i) an ABAQUS simulation of the wrinkling process, and (ii) an experiment on a $0.025 \mathrm{~mm}$ 


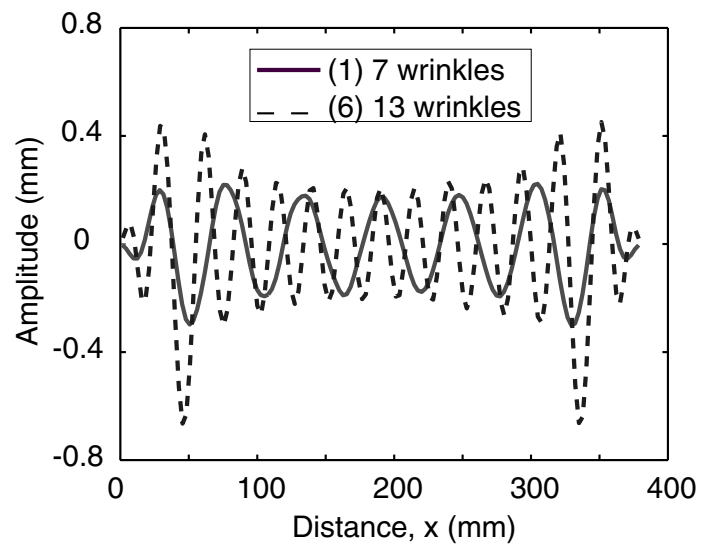

Figure 8. Wrinkle profiles from ABAQUS.

thick Kapton sheet mounted in a steel shear frame. The out-of-plane deflections of this membrane were measured with a non-contact device for monotonically increasing shear angles, both positive and negative. The smallest value of $\gamma$ for which measurements could be obtained was 0.00195 , i.e. $\delta=0.25 \mathrm{~mm}$.

For any given shear displacement, both the simulation and the experiment produce a characteristic pattern with two large side-wrinkles - corresponding to wrinkle $\mathrm{b}$ in Figure 3- and a series of wrinkles of approximately uniform amplitude in between. Figure 8 shows the crosssection of the membrane at mid-height, for the configurations immediately following jumps 1 and 6, defined in Figures 5 and 6 . Note that the distance between successive peaks in Figure 8 has to be divided by $\sqrt{2}$ to obtain the wrinkle wavelength. The side-wrinkles, which are only beginning to show after jump 1, are clearly visible after jump 6 .

After carrying out the simulation and experiment the average wrinkle wavelengths and amplitudes were determined after excluding the region of the edge wrinkles. Figures 9 and 10 show plots of these two parameters, together with the predictions from our theory; note that the experimental values plotted were obtained by averaging the values measured for positive and negative shear angles. Finally, Figure 11 compares the average, mid-plane, minor principal stress at mid-height of the membrane, predicted by ABAQUS, with predictions obtained by substituting Equation 10 into Equation 3.

It can be seen that the wavelengths obtained from ABAQUS and the experiment differ by less than $2 \mathrm{~mm}$ (note that the numbers on the ordinate of Figure 9 have to be doubled), i.e. about $12 \%$ of the values. 


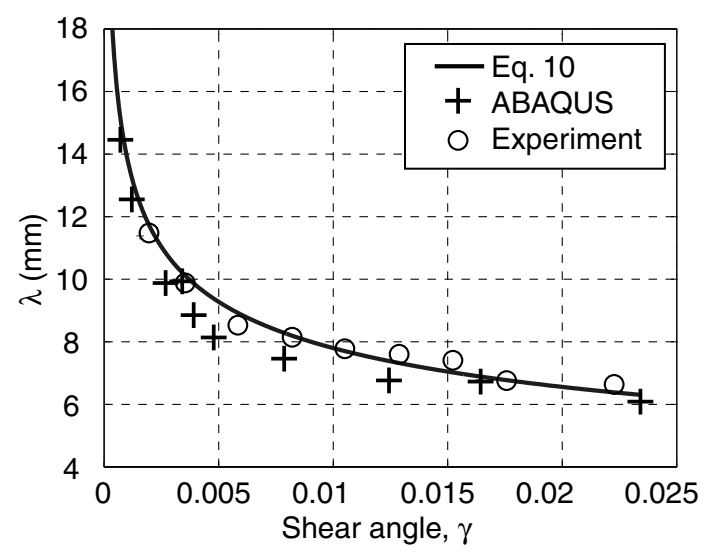

Figure 9. Comparison of wrinkle half-wavelengths.

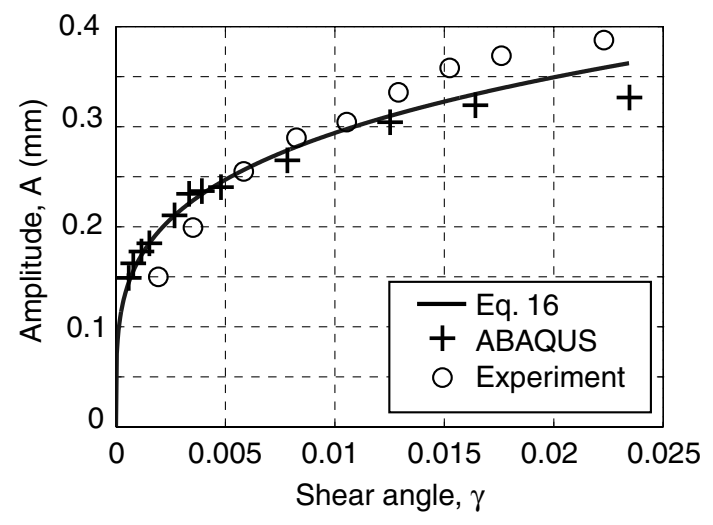

Figure 10. Comparison of wrinkle amplitudes.

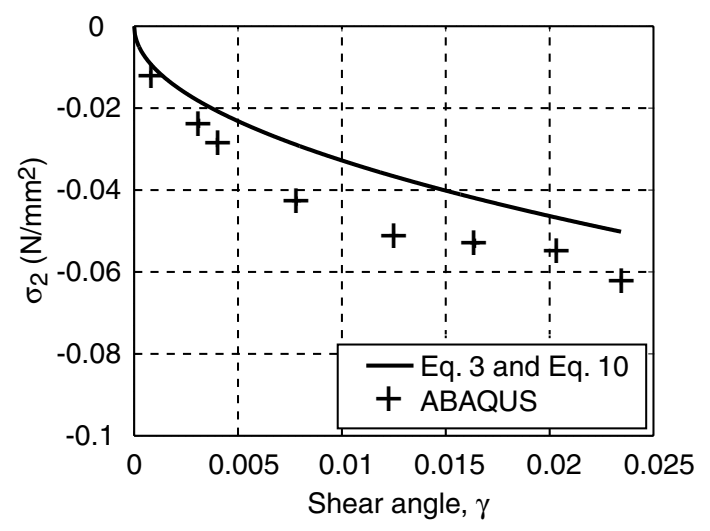

Figure 11. Comparison of compressive stress at mid-height. 
The theoretically predicted amplitudes follow both the experiment and the ABAQUS predictions very closely up to $\gamma \approx 1 \%$; the Kapton remains elastic within this range. For larger values of $\gamma$ some plastic deformation occurs; it is remarkable, and perhaps fortuitous, that in Figure 10 the theoretical predictions fall right in the middle of the range of the ABAQUS predictions and experimental measurements.

\section{DISCUSSION AND CONCLUSION}

It has been shown that the wrinkle half-wavelength, $\lambda$, of a membrane in simple shear is accurately predicted by Equation 10 and the amplitude, $A$, by Equation 16. Thus, $\lambda$ is inversely proportional and $A$ is directly proportional to the fourth root of the shear angle; and both of them are directly proportional to the square roots of the width and thickness of the membrane. They are both independent of the Young's Modulus. Therefore, in the sheared-panel problem the wrinkle amplitude varies quite slowly with the angle of shear.

The equilibrium formulation of our theory can be readily extended to membranes with other boundary conditions; it will be interesting to see if these conclusions remain valid. However, it should be noted that the assumed mode-shape allows the required geometric strain only in the middle of the membrane. A more accurate description of the wrinkled surface may be required, in future.

To conclude, we note that the rather large spread in our numerical and experimental results is due, in part at least, to the fact that the number of wrinkles corresponding to a particular shear angle depends on the exact number of bifurcations that have taken place, e.g. in Figure 6 , which is sensitive to small imperfections. This may explain the discrepancies between the two nominally identical experiments. The irregularities in the variation of the number of wrinkles with the shear angle originate from the difference between the response predicted upon increasing or decreasing the angle of shear, coupled with the fact that instead of using a path-following algorithm - we have used displacement incrementation.

\section{Acknowledgments}

We thank Chris Calladine for many useful discussions and for suggesting the approach presented in Section 3.1. Financial support for Y.W.W. from the Cambridge Commonwealth Trust is gratefully acknowledged. 


\section{References}

ABAQUS (2000) Theory and User's Manual Version 6.1. Hibbit Karlsson \& Sorensen Inc.

Adler, A. (2000) Finite Element Approaches for Static and Dynamic Analysis of Partially Wrinkled Membrane Structures. Ph.D. Dissertation, The University of Colorado at Boulder.

Calladine, C.R. (1983) Theory of Shell Structures, Cambridge University Press, Cambridge.

Epstein, M. and Forcinito, M.A. (2001) Anisotropic membrane wrinkling: theory and analysis. Int. J. Solids Structures 38, 5253-5272.

Jenkins, C. and Leonard, J.W. (1991) Nonlinear dynamic response of membranes: State of the art. ASME Appl. Mech. Reviews, 44, 319-328.

Liu, X., Jenkins, C.H. and Schur, W.W. (2000) Fine scale analysis of wrinkled membranes. Int. J. Computational Eng. Sci. 1, 281-298.

Mansfield, E.H. (1969) Tension field theory a new approach which shows its duality with inextensional theory. Proc. 12th Int. Cong. Appl. Mech., 305-320.

Mansfield, E.H. (1981) Gravity-induced wrinkle lines in vertical membranes. Proc. Roy. Soc. Lond. A 375, 307-325.

Mansfield, E.H. (1989) The Bending and Stretching of Plates, second edition, Cambridge University Press, Cambridge.

Pipkin, A.C. (1986) The relaxed energy density for isotropic elastic membranes. IMA J. Appl. Math., 36 85-99.

Reissner, E. (1938) On tension field theory. Proc. 5th Int. Cong. Appl. Mech., 88-92.

Rimrott, F.P.J. and Cvercko, M. (1985) Wrinkling in thin plates due to in-plane body forces. In: Inelastic Behaviour of Plates and Shells, (Edited by L. Bevilacqua, R. Feijoo and R. Valid), 19-48. Springer-Verlag.

Stein, M. and Hedgepeth, J.M. (1961). Analysis of Partly Wrinkled Membranes. NASA Langley Research Center, NASA TN D-813.

Wagner, H. (1929) Flat sheet metal girder with very thin metal web, Zeitschrift für Flugtechnik Motorlurftschiffahrt. 20, 200-207, 227-233, 256-262, 279-284.

Wong, Y.W. (2000) Analysis of Wrinkle Patterns in Prestressed Membrane Structures. M.Phil. Dissertation, University of Cambridge. 